\title{
The ameliorative effect of curcumin on cryptorchid and non-cryptorchid testes in induced unilateral cryptorchidism in albino rat: histological evaluation
}

\author{
M.A. Abd-El-Hafez'1, M.D. El-Shafee', S.H. Omar' ${ }^{1}$, A.A. Aburahma², S.S. Kamar'1(1) \\ ${ }^{1}$ Department of Medical Histology and Cell Biology, Faculty of Medicine, Cairo University, Cairo, Egypt \\ ${ }^{2}$ Department of Andrology, Faculty of Medicine, Cairo University, Cairo, Egypt
}

[Received: 17 May 2020; Accepted: 30 June 2020; Early publication date: 29 July 2020]

Background: Cryptorchidism, one or both hidden testes, is the most common abnormality of male sexual development. Subfertility or infertility is associated with both unilateral and bilateral cryptorchidism. In this study, we investigate the possible ameliorative effect of curcumin (Cur) on the induced-unilateral cryptorchidism testicular injury in both cryptorchid (Cryp) and non-cryptorchid (non-Cryp) scrotal testes through histological, immunohistochemical and morphometrics.

Materials and methods: Forty adult male albino rats were divided into: control group, Cur control group, Cryp group, and Cryp+Cur group. The rat model was surgically established by fixing the left testis in the abdomen. The treated groups were subjected to surgically induced-unilateral cryptorchidism on the left side then were given Cur (80 mg/kg) orally, for 20 days. Histological analysis using haematoxylin and eosin and periodic acid Schiff's reaction was done. Immunohistochemistry was performed for proliferating cell nuclear antigen (PCNA); to estimate the proliferation in the germinal epithelium, and vimentin; to evaluate Sertoli cells. The results were confirmed by statistical evaluation of the spermatogenic epithelium height, the seminiferous tubules diameter, the basement membrane thickness, the number of PCNA immunostained cells and the area per cent of vimentin immunostaining. Results: Distorted seminiferous tubules, substantial degeneration of the germinal epithelium, thickening of the basement membrane with a significant decrease in PCNA and vimentin immunostaining were observed in Cryp group; mainly in the cryptorchid testis. These structural changes were significantly reversed in Crypt+Cur group.

Conclusions: Curcumin proved to be an important and effective medical line for protecting against the unfavourable sequels of cryptorchidism in a rat model. (Folia Morphol 2021; 80, 3: 596-604)

Key words: testis, unilateral cryptorchidism, curcumin, proliferating cell nuclear antigen (PCNA), vimentin

Address for correspondence: Dr. S.S. Kamar, Department of Medical Histology and Cell Biology, Faculty of Medicine, Cairo University, Cairo, Egypt, tel: +20 01008909069, e-mail: Dr_samaakamar@yahoo.com

The work was conducted in: Faculty of Medicine, Cairo University, Cairo, Egypt.

This article is available in open access under Creative Common Attribution-Non-Commercial-No Derivatives 4.0 International (CC BY-NC-ND 4.0) license, allowing to download articles and share them with others as long as they credit the authors and the publisher, but without permission to change them in any way or use them commercially. 


\section{INTRODUCTION}

Cryptorchidism, one or both hidden testes, is the most common abnormality of male sexual development occurring in about $2.4-5 \%$ of full-term newborns [14]. The ratio rises to $30 \%$ of premature neonates. Related complications of infertility, malignant transformation, depression and trauma have been recorded [15].

Subfertility or infertility is associated with both unilateral and bilateral cryptorchidism. One-third of unilateral undescended testis complained of fertility impairment with incidence of azoospermia in about $13 \%$ of cases [11]. The impact of cryptorchidism included not only degeneration of germ cells in response to elevated temperature [17] but also increased intratesticular oxidative stress that produced deleterious testicular changes with reduced spermatogenesis [7]. A previous study has demonstrated that unilateral cryptorchidism was associated with increased number of mast cells in both testes, resulting in fibrosis and deterioration of spermatogenesis [1]. The structural defects of both the retained and scrotal testis have been concluded in other studies $[15,35]$.

The concept of 'early orchidopexy' has established as the primary treatment for cryptorchidism. However, orchidopexy alone is insufficient to completely restore spermatogenesis and there is a domain for a germinal epithelial protective substance [6]. Human spermatogonial from cryptorchid (Cryp) patients can piecemeally differentiate into haploid spermatids when treated with retinoic acid and stem cell factor [36]. Antioxidants have proved to significantly increase the sperm count and germ cell count in Cryp rats [3].

Curcumin (Cur), the active ingredient of the dietary spice turmeric (curcuma longa), is widely used in medical practice. Its efficacy is due to its phenolic group [33]. It is known to be anti-inflammatory, antineoplastic, cardioprotective and renoprotective reagent [36]. Moreover, it has bi-functional antioxidant effects by protecting the cell against the reactive species and stimulating up regulation of cytoprotective proteins [34].

The aim of our study is to evaluate the possible ameliorative effect of Cur on the induced unilateral cryptorchidism in both Cryp and non-Cryp testes.

\section{MATERIALS AND METHODS}

\section{Experimental design}

This study included 40 adult male albino rats, each of 200-250 $\mathrm{g}$ body weight. The animals were bred in the Animal House of Faculty of Medicine, Cairo University. Each group, or subgroup, was kept in separate wire cage at room temperature, fed ad libitum with free water supply. All procedures were held under ethical guidelines of Animal Care and Use Committee of Cairo University. The rats were divided equally into four groups $(n=10)$.

Control group (Gpl): the rats were equally subdivided into two subgroups $(n=5)$ : Blank control (Gpla): the rats were not exposed to any surgical procedure; and Sham (Gplb): subjected to sham operation on day 1 of the experiment. Under anaesthesia and complete aseptic conditions, a lower midline abdominal incision was performed. The left testis was displaced into the abdomen then replaced again into the scrotal sac, and then the incision was closed. The rats were administered buprenorphine $0.05 \mathrm{mg} / \mathrm{kg}$ by intraperitoneal injection $/ 8 \mathrm{~h}$; as post-operative analgesic for 7 days. Besides, they were given $1 \mathrm{~mL}$ dimethyl sulfoxide (DMSO) orally once daily for 20 days.

Curcumin (Cur) control group (GpII): were given Cur at a dose of $80 \mathrm{mg} / \mathrm{kg}$, dissolved in $1 \mathrm{mLDMSO}$, once daily orally, for 20 days.

Cryptorchid (Cryp) group (GpIII): rats were subjected to surgically induced-unilateral cryptorchidism on the left side. Animals were subjected to the same surgical procedures as Gplb but the left testis was displaced into the abdomen and fixed. The gubernaculum on the left side was separated, the testis was displaced into the abdomen, and the inguinal canal was closed [7]. The incision was sutured and the rats were administered post-operative analgesic. The animals were left for 20 days after surgery without any treatment [22].

Cryptorchid plus curcumin (Cryp+Cur) group (GpIV): rats were subjected to surgically induced-unilateral cryptorchidism on the left side as described in GpllI then were given Cur orally, for 20 days.

All rats were euthanized at the end of the experiment by intraperitoneal injection of thiopental sodium $(50 \mathrm{mg} / \mathrm{kg})$. Testis specimens were fixed in Bouin solution and embedded in paraffin. Serial sections of $5 \mu \mathrm{m}$ thickness were cut and subjected to histological and immunohistochemical studies.

\section{Histological studies}

Haematoxylin and eosin stain (HE) to illustrate the morphological change and periodic acid Schiff's (PAS) reaction to demonstrate the basement membrane.

Immunohistochemistry for proliferating cell nuclear antigen, using a mouse monoclonal antibody (Ab) 
(Thermo Scientific Laboratories, USA, Cat.\# MS106P) as a criterion for the proliferating cells.

Vimentin intermediate filament, using monoclonal antibody (Cell Marque Corporation, Toll-Free North America, Cat.\# 347M-18).

\section{Morphometrics}

Employing "Leica-Qwin $500 \mathrm{C}$ " image analyser (Cambridge, England), 10 non-overlapping fields/rat testes were examined and the following parameters were estimated:

- height of the spermatogenic epithelium (SEp) and the diameter of the seminiferous tubules in HE sections;

- thickness of the basement membrane in PAS stained sections;

- number of positive (+ve) proliferating cell nuclear antigen (PCNA) immunostained cells;

- area per cent of + ve vimentin immunostaining.

\section{Statistical studies}

The estimated measurements were compared and analysed using one-way analysis of variance of SPSS software version-19. Comparison between the different groups was followed by post-hoc Tukey test. Quantitative representative data was obtained and summarised as means \pm standard deviations (SD). Probability ( $p$ ) values $<0.05$ were considered statistically significant.

\section{RESULTS}

Clinical observation. No mortality was recorded in the experimental rats and no changes were noted in their behaviours in water and food consumption.

HE stain. Testicular sections of control rats and rats from Gpll displayed normal architecture of the seminiferous tubules and the interstitial tissue (Fig. 1A, B). The Cryp testes of Gplll revealed severely distorted seminiferous tubules and partial separation of the basement membrane in some areas. In most of the examined fields, there was obvious degeneration of the germinal epithelium with some shed cells in the lumen. The non-Cryp testes from Gplll showed mild disorganisation of the seminiferous tubules. The lining epithelium showed spermatogonia, primary spermatocytes with absence of late stage of germ cells (Fig. 1C, D). The GpIV revealed obvious protection of the abdominal and scrotal testes. There were normal structure of the seminiferous tubules containing spermatozoa in the lumen and the interstitial tissue in both abdominal and scrotal testes. Seminiferous tubule displayed all germinal cell layers with sperm in the lumen (Fig. 1E, F).

PAS stain. Sections from Gplll revealed strong thick + ve PAS reaction in the thick irregular basement membrane in both scrotal and abdominal testes. In GpIV, thin strong + ve PAS reaction in the basement membrane of both abdominal and scrotal testes was illustrated (Fig. 2).

Immunohistochemistry (Fig. 3). Using PCNA immunostaining, the Cryp testes from Gplll showed few +ve PCNA immunostained cells near the basement membrane in severely degenerated SEp. The non-Cryp testes revealed many + ve PCNA immunostained cells in the early stages of the SEp and weak or absent immunostaining in the late stages of the spermatogenic cells. GpIV revealed diffuse + ve PCNA immunostaining in both testes.

In vimentin immunohistochemistry, Cryp testes of Gplll showed + ve vimentin immunostaining in Sertoli cells in the perinuclear region. The right scrotal testes of the same rats revealed + ve vimentin immunostaining mainly in the perinuclear region of Sertoli cells with few + ve apical immunostaining. In GpIV, both testes revealed numerous + ve vimentin immunostaining of Sertoli cells in the perinuclear regions and throughout the cytoplasm extending into the apices.

\section{Statistical analysis (Table 1)}

The height of the SEp and seminiferous tubules diameter. Gplll showed a significant decrease in both parameters as compared to Gpl, Gpll, and GpIV. Meanwhile, both parameters in the Cryp testes of Gplll were significantly decreased as compared to the scrotal testis of GpIII. In GpIV, the mean height of SEp and the mean diameter of seminiferous tubules in the Cryp testes were significantly decreased as compared to Gpl, Gpll and non-Cryp testes of GpIV.

The thickness of the basement membrane. In both testes of GpllI, the mean thickness of the basement membrane displayed a significant increase as compared with Gpl, Gpll, and GpIV. In addition, the mean thickness of basement membrane in Cryp testes of Gplll was significantly increased compared to the non-Cryp testes of Gplll. The mean thickness of basement membrane in Cryp and non-Cryp testes of GpIV were comparable.

The mean cell count of + ve PCNA immunostained cells. In both testes of Gplll, the mean cell count of + ve PCNA immunostained cell was significantly decreased as compared to Gpl, Gpll and 


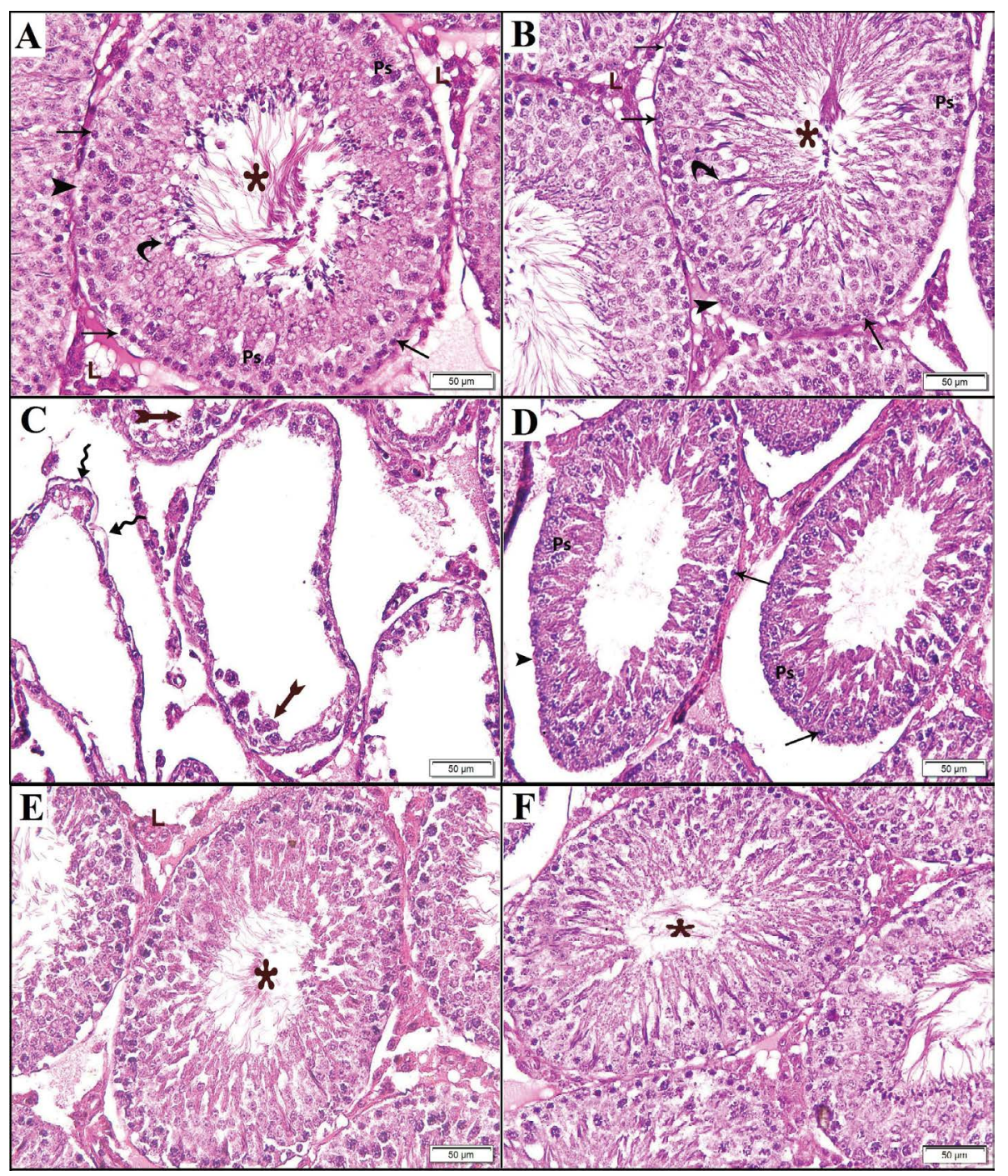

Figure 1. Haematoxylin and eosin (HE)-stained rat testes $(\times 200)$ showing Group I (Gpl; A) and Group II (Gpll; B): the seminiferous tubules containing spermatozoa (star) in the lumen, the interstitial tissue (IS) harbouring clusters of Leydig's cells (L), the spermatogenic epithelium (SEp) resting on basal lamina with Sertoli cells (arrowheads), attached mature spermatids (curved arrows), numerous spermatogonia (arrows) and primary spermatocytes (Ps); C. Cryptorchid testis in Group III (GpllI): substantial distortion and collapse of the seminiferous tubules with partial separation of the basement membrane (wavy-arrow), obvious degeneration of the germinal epithelium. Some cells are sloughed in the lumen (bifid-arrow); D. Non-cryptorchid testis in Gplll: mild disorganisation of the seminiferous tubules with expansion of interstitial space, the SEp illustrating Sertoli cells (arrowhead), spermatogonia (arrows), Ps, and absence of sperms; Cryptorchid and (E) and non-cryptorchid testes (F) in Group IV (GpIV): normal structure of the seminiferous tubules with all germinal cell layers, mature spermatids and spermatozoa (star) in the lumen, and IS containing clusters of Leydig's cells (L).

GpIV. Besides, it was significantly decreased in the Cryp testes of Gplll as compared to the non-Cryp testes of Gplll. The mean cell count of + ve PCNA immunostained cell in the Cryp testes of GpIV was significantly decreased as compared with Gpl, Gpll and non-Cryp testis of GpIV.

The mean area per cent of vimentin immunostaining. Both testis of Gplll showed a significant decrease as compared to Gpll and GpIV. In the abdominal testes of GpIV, it was significantly decreased as compared to Gpl, Gpll and scrotal testes of GpIV.

\section{DISCUSSION}

Cryptorchidism is a common congenital malformation in the male reproductive system. It is documented to have long-term sequels such as infertility, depression, and testicular cancer. The impacts included degeneration of germ cells in response to elevated temperature [17] and increased intratesticular oxidative stress [7]. The objectives for cryptorchidism management are to preserve fertility and ameliorate the risk of malignancy [29].

Experimentally induced unilateral cryptorchidism is stellar method to study undescended testis in 


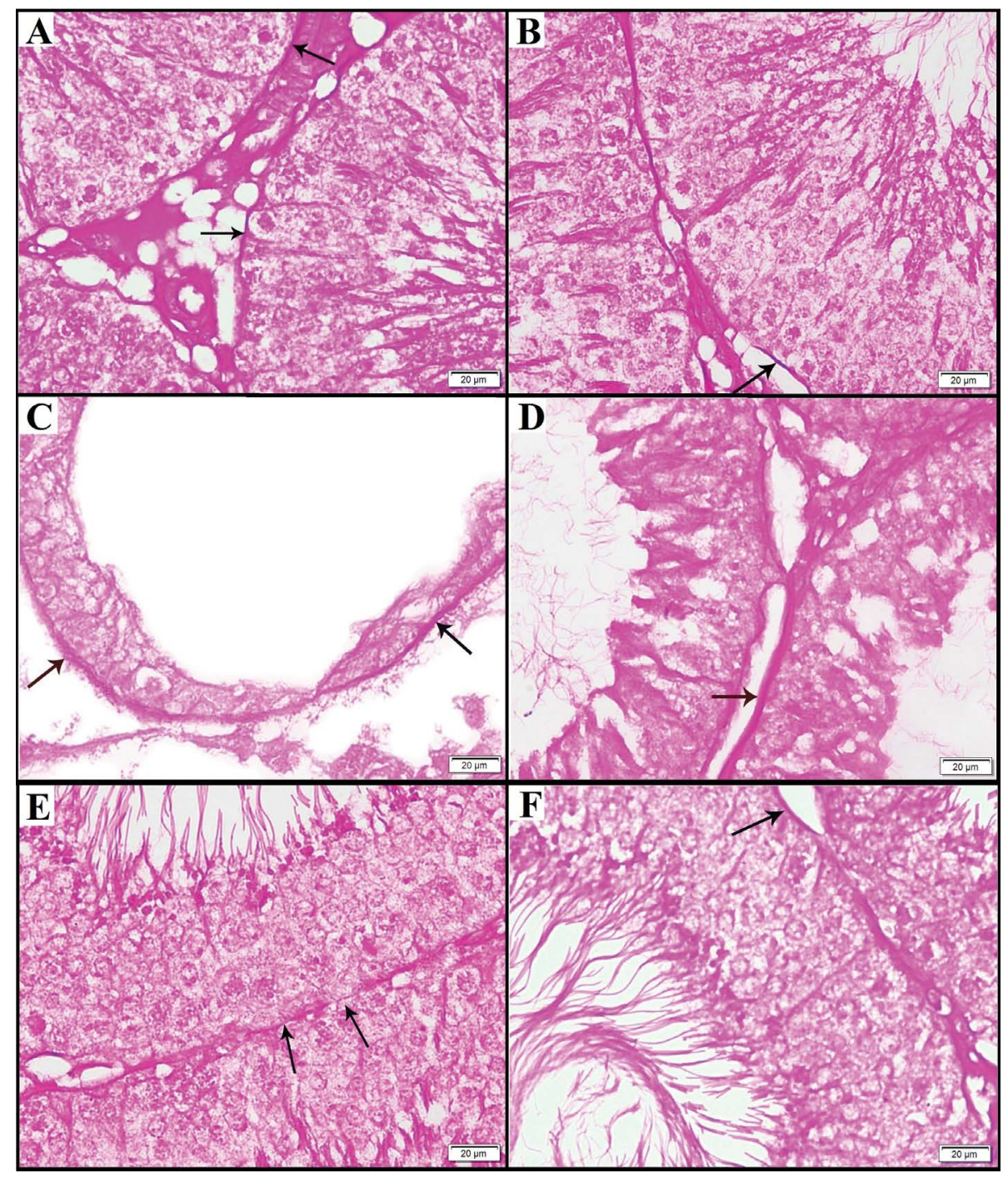

Figure 2. Periodic acid Schiff's (PAS)-stained rat testes $(\times 400)$ showing: Group I (Gpl; A) and Group II (Gpll; B): thin strong + ve PAS reaction in the basement membrane (arrows); Cryptorchid (C) and non-cryptorchid (D) testes in Group III (GpllI): strong + ve PAS reaction in the thick irregular basement membrane (arrows). Cryptorchid (E) and non-cryptorchid (F) testes in Group IV (GpIV): thin strong + ve PAS reaction in the basement membrane (arrows).

relevance to spermatogenesis against temperature gradient in both testes [12]. In the present work, cryptorchid testes of Gplll revealed severely distorted seminiferous tubules and SEp. The non-Cryp testes of Gplll displayed mild disorganisation of the seminiferous tubules with absence of sperm in most of fields. However, a significant difference in the height of germinal epithelium and the diameter of seminiferous tubules was noted between the Cryp and the non-Cryp testes of Gplll. These findings were in accordance with previous study of Moon et al. [25]. It was postulated that histological changes associated with cryptorchidism resulted in a significant reduction in the number and the diameter of seminiferous tubules with amelioration of the number and prolif- eration of spermatogonia. Besides, most of the proliferating cells detected were Sertoli cells suggesting increased risk of Sertoli cell tumours. Also, structural defects of both the retained and scrotal testes were reported [4, 35].

High temperature was suggested to induce disruption of spermatogenesis in cryptorchid testis [12]. Lin et al. [20] found that hyperthermia initiates oxidative stress and apoptosis in spermatogenic cells with subsequent affection of fertility. This was explained by Tekayev et al. [32] that testicular tissues are rich in polyunsaturated fatty acids and poor in antioxidant defence. Thus, they are prone to be attacked by reactive oxygen species (ROS) which are able to oxidize proteins, lipids and deoxyribonucleic acid leading to 


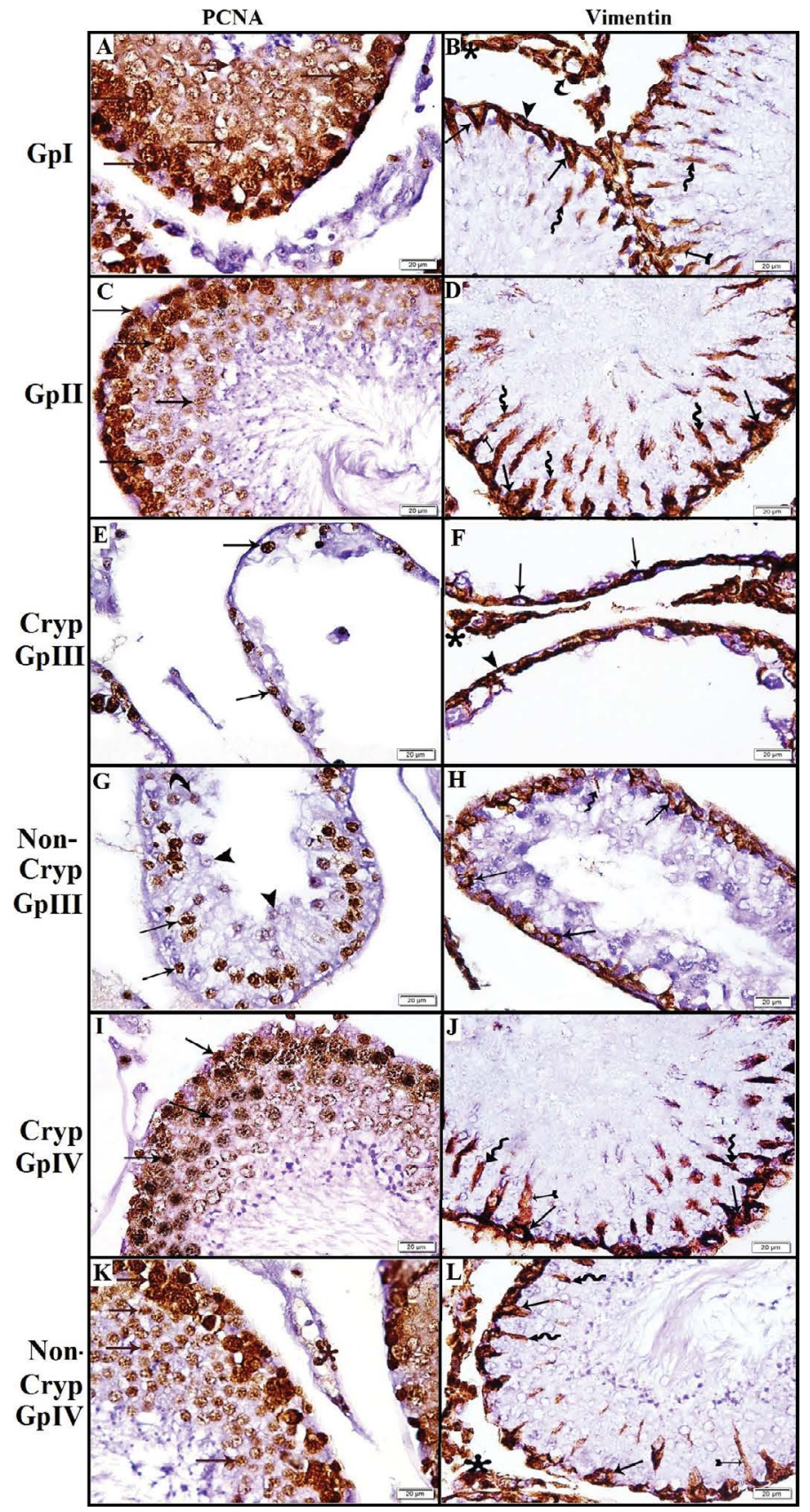

Figure 3. Proliferating cell nuclear antigen (PCNA) and vimentin immunohistochemically stained rat testes $(\times 400)$. PCNA immunohistochemistry showing: Group I (Gpl; A) and Group II (Gpll; C): diffuse + ve PCNA immunostaining in the nuclei of spermatogenic cells (arrow) and in the interstitial tissue (IS) (star); E. Cryptorchid testis in Group III (GpllI): few + ve PCNA immunostained cells (arrow) near the basement membrane in severely degenerated spermatogenic epithelium (SEp); G. Non-cryptorchid testis in Group III (GpIIII): many PCNA immunostained cells in the early stages of the spermatogenic cells (arrow) with weak (curved-arrow) or absent (arrowhead) immunostaining in the late stages of the spermatogenic cells; Cryptorchid (I) and (K) non-cryptorchid testes in Group IV (GpIV): diffuse + ve PCNA immunostaining in the nuclei of spermatogenic cells (arrow). Vimentin immunohistochemistry showing: Group I (Gpl; B) and Group II (Gpll; D): + ve vimentin immunostaining of Sertoli cells in the perinuclear region (arrows), throughout the cytoplasm (bifid-arrow) and apically from nucleus (wavy-arrows). Positive immunostaining is noted in myoid cells (arrowhead), endothelium (curved-arrow) and IS cells (star); F. Cryptorchid testis in Gplll: + ve vimentin immunostaining around the nuclei of Sertoli cells (arrows), in myoid cells (arrowhead) and IS cells (star); H. Non-cryptorchid testis in Gplll: + ve vimentin immunostaining mainly in the perinuclear region of Sertoli cells (arrows) with few + ve immunostaining extending through the cytoplasm apically (wavy-arrow); Cryptorchid (J) and non-cryptorchid (L) testes in GplV: + ve vimentin immunostaining of Sertoli cells in the perinuclear region (arrows), throughout the cytoplasm (bifid-arrow) and apically from nucleus (wavy-arrows); Cryp - cryptorchid.

Table 1. Curcumin significantly protected the testicular tissue and improved the germinal epithelium proliferation in both cryptorchid (Cryp) and non-cryptorchid testes

\begin{tabular}{lccccc}
\hline Groups & $\begin{array}{c}\text { Mean height } \\
\text { of spermatogenic } \\
\text { epithelium }[\mu \mathrm{m}]\end{array}$ & $\begin{array}{c}\text { Mean diameter } \\
\text { of seminiferous } \\
\text { tubule }[\mu \mathrm{m}]\end{array}$ & $\begin{array}{c}\text { Mean diameter of the } \\
\text { basement membrane } \\
\text { thickness }[\mu \mathrm{m}]\end{array}$ & $\begin{array}{c}\text { Mean cell count } \\
\text { of PCNA immunos- } \\
\text { tained cells }\end{array}$ & $\begin{array}{c}\text { Mean area per } \\
\text { cent of vimentin } \\
\text { immunostaining }\end{array}$ \\
\hline Group I & $97.01 \pm 5.64$ & $362.27 \pm 16.48$ & $0.85 \pm 0.15$ & $36.7 \pm 2.93$ & $26.2 \pm 3$ \\
Group II & $97 \pm 5.47$ & $357.29 \pm 10.13$ & $0.9 \pm 0.13$ & $38.1 \pm 2.92$ & $26.23 \pm 2.66$ \\
Cryp; Group III & $9.18 \pm 2.25^{*} ! \#$ & $141.94 \pm 14.62^{*} ! \#$ & $5.61 \pm 0.61^{*} ! \#$ & $6.7 \pm 1.49^{*} ! \#$ & $10.89 \pm 2.09^{*} \#$ \\
Non-Cryp; Group III & $69.87 \pm 7.49^{*} \#$ & $202.93 \pm 23.96^{*} \#$ & $4.67 \pm 0.72^{*} \#$ & $11.6 \pm 2.01^{*} \#$ & $11.51 \pm 1.76^{*} \#$ \\
Cryp; Group IV & $89.29 \pm 2.38^{*} \$$ & $297.12 \pm 14.99^{*} \$$ & $1.13 \pm 0.32$ & $32.1 \pm 2.08^{*} \$$ & $20.28 \pm 2.37^{*} \$$ \\
Non-Cryp; Group IV & $95.90 \pm 3.89$ & $348.62 \pm 14.58$ & $0.84 \pm 0.15$ & $36.6 \pm 2.07$ & $24.3 \pm 2.05$ \\
\hline
\end{tabular}

Data presented with means \pm standard deviation. *Significant as compared to Group I; ! Significant as compared to Cryp Group III; \#Significant as compared to Cryp and non-Cryp group IV; $\$$ Significant as compared to non-Cryp Group IV; PCNA — proliferating cell nuclear antigen 
cellular damage. The equalisation between production and clearance of ROS provides an important role in the spermatogenesis as physiological level of ROS maintains the body's normal physiological functions, whereas excessive ROS can cause apoptosis.

Interestingly, Acikgoz et al. [1] found that unilateral cryptorchidism was associated with increased number of mast cells in both testes, resulting in deterioration of spermatogenesis. According Aydin et al. [5], the unilateral cryptorchidism causes endocrine dysfunction in the body, influencing the secretion of sex hormone and occurrence of allergic reaction. Recent study has revealed that cryptorchidism leads to hypothalamic-pituitary-gonadal dysfunction, which was assumed to interfere with the contralateral testicular function and morphology [30]. Moreover, it was documented that affection of sensory branch of genito-femoral nerve is a finding in cryptorchidism [17]. The abnormal environment in the Cryp testis has deleterious effects on the genito-femoral nerve that induces changes in blood circulation and the microenvironment in the contralateral non-Cryp testis [26].

Rats from GpIV that received curcumin treatment after induction of unilateral cryptorchidism revealed obvious protection of the abdominal and scrotal testes. They exhibited normal architecture of the seminiferous tubules and SEp. This result was approved by the significant increase in the mean height of germinal epithelium and the mean diameter of the seminiferous tubules as compared to Gplll. However, the means in the non-Cryp testes of GpIV was comparable to $\mathrm{Gpl}$ and significantly increased as compared to the Cryp testes of GpIV. Thus, indicating substantial protective effect of Cur in the Cryp testis and full protection in the contralateral non-Cryp testis.

In the current study, Gplll displayed significantly thickened irregular basement membrane in both testes as compared to Gpl. However, GpIV revealed thin basement membrane of both testes that was comparable to Gpl. In the study of Hassanin et al. [16], the thickening of the basement membrane was detected and explained by the harmful effect of oxidative stress on the testis induced by acrylamide. The protective effect of Cur could be referred to its antioxidant effect similar to the antioxidant vitamin $\mathrm{E}$ that protected the testis from the oxidative stress.

In PCNA immunohistochemistry, control rats showed diffuse nuclear immunostaining in the spermatogenic cells in addition to the interstitial tissue.
Thus, indicating healthy high proliferative capacity of the testicular tissue [28]. In Gplll, significant decrease in PCNA immunostained cells was noted as compared to Gpl. If any in the cryptorcid testes, the PCNA immunostained cells were noted near the basement membrane. The scrotal testes showed + ve PCNA immunostained cells, mainly in the early stages of the spermatogenic cells and weak or absent PCNA immunostaining in the late stages. It was reported that the testicular tissues obtained from rats treated with cadmium had a harmful effect on the testis through its oxidative stress activity [27]. This was supported by Dutta et al. [12] who proved that oxidative stress ameliorate the proliferation and induces apoptosis in the highly differentiated spermatogenic cells causing its degeneration in the order of; sperms, spermatids, spermatocytes then spermatogonia.

Testicular sections of rats from GpIV revealed substantial + ve PCNA immunostaining in both abdominal and scrotal testes. However, this significant preservation of the proliferative capacity was still partial in the Cryp testes of GpIV as compared to the scrotal testes of the same group. This is in agreement with Yang et al. [37] who reported that human spermatogonial stem cells from cryptorchid patients can progressively differentiate into haploid spermatids when treated with the antioxidant retinoic acid and stem cell factor. Previous studies proved that antioxidants significantly increase the sperm count and germ cell count in cryptorchid rats [2,3].

In addition to antioxidant role, it was indicated that curcumin enhances the proliferation, stemness and colony formation in dose dependent manner. In small doses, it increased the expression of marker proteins coupled with the cell growth, telomerase activity and stemness acting signalling pathways [19]. Furthermore, it was reported that curcumin is a favourable anticancer drug due to its beneficial induction of proliferation arrest and cell death in a variety of tumour cells through down-regulation of specific proteins [18]. According to the study of Cao et al. [8] Cur inhibited cancer cell proliferation and augmented apoptosis of osteoclastoma cells via repression of matrix metalloproteinase- 9 and nuclear factor kappa beta, and stimulation of c-Jun $\mathrm{N}$-terminal kinases signalling pathways. In addition to the study of Srivastava et al. [31], it synergistically modulated Wnt/beta-catenin signalling pathways and possessed anti-proliferative activity in multi- 
ple cancer cell lines. All these findings support the advantageous use of Cur in cryptorchidism, not only to enhance the stemness and proliferation of spermatogonia but also to protect against cancer development.

Vimentin immunohistochemistry in control rats illustrated +ve immunostaining of Sertoli cells basally with characteristic apical projections. Vimentin is an intermediate filament detected in mature Sertoli cells. Its distribution pattern is harmonic with its pivotal role in maintaining tissue integrity and preservation of spermatogenesis. It radiates apically in the cytoplasm to become attached with the specialized membrane junctions, desmosome-like junctions that connect germ cells with Sertoli cells [24].

Cryptorchid testes of Gplll showed substantial reduction in the vimentin immunostaining; mostly detected basally around nuclei of Sertoli cells indicating collapse of the vimentin intermediate filaments and their disorganization in the basal region of the Sertoli cells. The scrotal testes of the same rats revealed few apical vimentin immunostaining besides the perinuclear region denoting partial separation of vimentin away from the plasma membrane [13]. In agreement, Mohammed et al. [23] detected significant decrease of vimentin immunostaining in astaxanthin-induced-testicular damage. The damage was triggered by chronic stress through excessive production of free radicals.

It was reported that cytoskeleton, adherence proteins and cellular adhesion molecules functionally work inter-dependently rather than independently in the homeostasis of spermatogenic cellular junctions [10]. Besides, the disrupted inter-Sertoli germ cell junctions have been demonstrated to cross talk with the defective spermatogenesis in cryptorchidism [9]. On the other hand, the anchoring junction proteins are involved in the regulation of germ cell apoptosis. They can disrupt vimentin filaments at the site of Sertoli germ cell anchoring junction, thus inducing up-regulation surge of the testicular Fas-receptor with subsequent germ cell apoptosis [38].

In GpIV, the mean area per cent of vimentin immunostaining was significantly increased as compared to Gplll. However, the Cryp testes of GpIV displayed significant difference as compared to $\mathrm{Gpl}$ and scrotal testes of GpIV. These findings signalize that curcumin is capable of preventing disaggregation of Sertoli germ cells contacts and spermatogenic cell apoptosis that was induced by hyperthermia [21].

\section{Limitations of the study}

The limitations of this work were the genetic and the hormonal factors that should be included in the estimation of curcumin on the unilateral cryptorchidism for upcoming clinical trials.

\section{CONCLUSIONS}

In conclusion, curcumin proved to be an important and effective medical line for protecting against the unfavourable sequels of cryptorchidism parallel to orcheopexy. It is one of the antioxidants that improve the fertility after surgery with potential role to protect against cancer transformation.

\section{Conflict of interest: None declared}

\section{REFERENCES}

1. Acikgoz A, Asci R, Aydin O, et al. The role of ketotifen in the prevention of testicular damage in rats with experimental unilateral undescended testes. Drug Des Devel Ther. 2014; 8: 2089-2097, doi: 10.2147/DDDT.S67941, indexed in Pubmed: 25364234.

2. Afeefy AA, Salah M, Amina MA. The role of vitamin $E$ in reducing aluminum hydroxide effects on testes of albino rats: a histological and immunohistochemical study. IRJABS. 2016; 10(4): 369-379, doi: 10.7860/ JCDR/2017/23927.9886.

3. Asadi N, Bahmani M, Kheradmand A, et al. The Impact of Oxidative Stress on Testicular Function and the Role of Antioxidants in Improving it: A Review. J Clin Diagn Res. 2017; 11(5): IE01-IE05, doi: 10.7860/JCDR/2017/23927.9886, indexed in Pubmed: 28658802.

4. Ateş F, Soydan H, Okçelik S, et al. Clinical and histopathological results of the adult patients with unilateral cryptorchidism. Turk J Urol. 2016; 42(2): 74-79, doi: 10.5152/ tud.2016.47886, indexed in Pubmed: 27274891.

5. Aydin S, Baykus Y, Deniz R. Human chorionic gonadotropin levels of pregnant women can be an indicator of boys with cryptorchidism. Med Hypotheses. 2018; 114: 18, doi: 10.1016/j.mehy.2018.02.035, indexed in Pubmed: 29602455.

6. Azari O, Gholipour H, Kheirandish R, et al. Study of the protective effect of vitamin $C$ on testicular tissue following experimental unilateral cryptorchidism in rats. Andrologia. 2014; 46(5): 495-503, doi: 10.1111/and.12106, indexed in Pubmed: 23635089.

7. Bae WJ, Ha US, Choi JB, et al. Protective effect of decursin extracted from angelica gigas in male infertility via Nrf2/HO-1 signaling pathway. Oxid Med Cell Longev. 2016; 2016: 5901098, doi: 10.1155/2016/5901098, indexed in Pubmed: 27034737.

8. Cao F, Liu T, Xu Y, et al. Curcumin inhibits cell proliferation and promotes apoptosis in human osteoclastoma cell through MMP-9, NF-kB and JNK signaling pathways. Int J Clin Exp Pathol. 2015; 8(6): 6037-6045, indexed in Pubmed: 26261481.

9. Chen J, Fok KL, Chen H, et al. Cryptorchidism-induced CFTR down-regulation results in disruption of testicular tight junctions through up-regulation of NF-kB/COX-2/PGE2. Hum Reprod. 2012; 27(9): 2585-2597, doi: 10.1093/ humrep/des254, indexed in Pubmed: 22777528. 
10. Cheng CY, Mruk DD. The blood-testis barrier and its implications for male contraception. Pharmacol Rev. 2012; 64(1): 16-64, doi: 10.1124/pr.110.002790, indexed in Pubmed: 22039149.

11. Chung E, Brock GB. Cryptorchidism and its impact on male fertility: a state of art review of current literature. Can Urol Assoc J. 2011; 5(3): 210-214, doi: 10.5489/cuaj.10106, indexed in Pubmed: 21672488.

12. Dutta $S$, Joshi KR, Sengupta $P$, et al. Unilateral and bilateral cryptorchidism and its effect on the testicular morphology, histology, accessory sex organs, and sperm count in laboratory mice. J Hum Reprod Sci. 2013; 6(2): 106-110, doi: 10.4103/0974-1208.117172, indexed in Pubmed: 24082651.

13. ElGhamrawy TA, Helmy D, Elall HFA. Cadherin and vimentin immunoexpression in the testis of normal and induced infertility models of albino rats. Folia Morphol. 2014; 73(3): 339-346, doi: 10.5603/FM.2014.0050, indexed in Pubmed: 25343410.

14. Fawzy F, Hussein A, Eid MM, et al. Cryptorchidism and Fertility. Clin Med Insights Reprod Health. 2015; 9: 39-43, doi: 10.4137/CMRH.S25056, indexed in Pubmed: 26740750.

15. Goel P, Rawat JD, Wakhlu A, et al. Undescended testicle: An update on fertility in cryptorchid men. Indian J Med Res. 2015; 141(2): 163-171, doi: 10.4103/0971-5916.155544, indexed in Pubmed: 25900951.

16. Hasanin NA, Sayed NM, Ghoneim FM, et al. Histological and Ultrastructure Study of the Testes of Acrylamide Exposed Adult Male Albino Rat and Evaluation of the Possible Protective Effect of Vitamin E Intake. J Microsc Ultrastruct. 2018; 6(1): 23-34, doi: 10.4103/JMAU.JMAU_7_18, indexed in Pubmed: 30023264.

17. Hutson JM, Li R, Southwell BR, et al. Germ cell development in the postnatal testis: the key to prevent malignancy in cryptorchidism? Front Endocrinol (Lausanne). 2012; 3: 176, doi: 10.3389/fendo.2012.00176, indexed in Pubmed: 23316184.

18. Jutooru I, Chadalapaka G, Lei P, et al. Inhibition of NFkappaB and pancreatic cancer cell and tumor growth by curcumin is dependent on specificity protein down-regulation. J Biol Chem. 2010; 285(33): 25332-25344, doi: 10.1074/jbc. M109.095240, indexed in Pubmed: 20538607.

19. Kim JH, Park SH, Nam SW, et al. Curcumin stimulates proliferation, stemness acting signals and migration of 3T3-L1 preadipocytes. Int J Mol Med. 2011; 28(3): 429-435, doi: 10.3892/ijmm.2011.680, indexed in Pubmed: 21519785.

20. Lin C, Choi YS, Park SGi, et al. Enhanced protective effects of combined treatment with beta-carotene and curcumin against hyperthermic spermatogenic disorders in mice. Biomed Res Int. 2016; 2016: 2572073, doi: 10.1155/2016/2572073, indexed in Pubmed: 28050551.

21. Lin C, Shin DG, Park SGi, et al. Curcumin dose-dependently improves spermatogenic disorders induced by scrotal heat stress in mice. Food Funct. 2015; 6(12): 3770-3777, doi: 10.1039/c5fo00726g, indexed in Pubmed: 26412282.

22. Liu F, Huang $H, X u Z L$, et al. Germ cell removal after induction of cryptorchidism in adult rats. Tissue Cell. 2012; 44(5): 281-287, doi: 10.1016/j.tice.2012.04.005, indexed in Pubmed: 22832057.

23. Mohammed M, Elfadeel KA, Abdel-Aziz $\mathrm{H}$, et al. The effect of chronic stress on the testis of adult albino Rats and the possible protective effect of astaxanthin supplementation (Histological, immunohistochemical and biochemical studies). J Med Histol. 2019; 2(2): 115-130, doi: 10.21608/ jmh.2019.7416.1048.

24. Monleón E, Noya A, Carmen Garza Ma, et al. Effects of an anti-gonadotrophin releasing hormone vaccine on the morphology, structure and function of bull testes. Theriogenology. 2020; 141: 211-218, doi: 10.1016/j.theriogenology.2019.07.019, indexed in Pubmed: 31387698.

25. Moon JH, Yoo DY, Jo YK, et al. Unilateral cryptorchidism induces morphological changes of testes and hyperplasia of Sertoli cells in a dog. Lab Anim Res. 2014; 30(4): 185-189, doi: 10.5625/lar.2014.30.4.185, indexed in Pubmed: 25628730.

26. Morandi G, Cerbone M, Lamback EB, et al. $45, X / 46$, XY mosaicism presenting with isolated unilateral cryptorchidism and a normal blood karyotype. J Clin Endocrinol Metab. 2018; 103(6): 2079-2082, doi: 10.1210/jc.2017-02671, indexed in Pubmed: 29618062.

27. Morsi AA, Shawky LM, El Bana EA. The potential gonadoprotective effects of grape seed extract against the histopathological alterations elicited in an animal model of cadmium-induced testicular toxicity. Folia Morphol. 2020; 79(4): 767-776, doi: 10.5603/FM.a2020.0003, indexed in Pubmed: 31930466.

28. Portela JMD, Heckmann L, Wistuba J, et al. Development and disease-dependent dynamics of spermatogonial subpopulations in human testicular tissues. J Clin Med. 2020; 9(1): 224, doi: 10.3390/jcm9010224, indexed in Pubmed: 31947706.

29. Radmayr C, Dogan HS, Hoebeke P, et al. Management of undescended testes: European Association of Urology/ European Society for Paediatric Urology Guidelines. J Pediatr Urol. 2016; 12(6): 335-343, doi: 10.1016/j. jpurol.2016.07.014, indexed in Pubmed: 27687532.

30. RodprasertW, Virtanen HE, Mäkelä JA, et al. Hypogonadism and Cryptorchidism. Front Endocrinol (Lausanne). 2019; 10: 906, doi: 10.3389/fendo.2019.00906, indexed in Pubmed: 32010061.

31. Srivastava NS, Srivastava RA. Curcumin and quercetin synergistically inhibit cancer cell proliferation in multiple cancer cells and modulate Wnt/ $\beta$-catenin signaling and apoptotic pathways in A375 cells. Phytomedicine. 2019; 52: 117-128, doi: 10.1016/j. phymed.2018.09.224, indexed in Pubmed: 30599890.

32. Tekayev M, Bostancieri N, Saadat KA, et al. Effects of Moringa oleifera Lam Extract (MOLE) in the heat shock protein 70 expression and germ cell apoptosis on experimentally induced cryptorchid testes of rats. Gene. 2019; 688: 140-150, doi: 10.1016/j.gene.2018.11.091, indexed in Pubmed: 30529510.

33. Trujillo J, Chirino YI, Molina-Jijón E, et al. Renoprotective effect of the antioxidant curcumin: Recent findings. Redox Biol. 2013; 1: 448-456, doi: 10.1016/j.redox.2013.09.003, indexed in Pubmed: 24191240.

34. Trujillo J, Granados-Castro LF, Zazueta C, et al. Mitochondria as a target in the therapeutic properties of curcumin. Arch Pharm (Weinheim). 2014; 347(12): 873-884, doi: 10.1002/ ardp.201400266, indexed in Pubmed: 25243820.

35. Tsounapi $P$, Honda M, Dimitriadis F, et al. Post-fertilization effect of bilateral primary testicular damage induced by unilateral cryptorchidism in the rat model. Andrology. 2016; 4(2): 297-305, doi: 10.1111/andr.12154, indexed in Pubmed: 26757429.

36. Ueki M, Ueno $\mathrm{M}$, Morishita J, et al. Curcumin ameliorates cisplatin-induced nephrotoxicity by inhibiting renal inflammation in mice. J Biosci Bioeng. 2013; 115(5): 547-551, doi: 10.1016/j.jbiosc.2012.11.007, indexed in Pubmed: 23245727.

37. Yang S, Ping P, Ma M, et al. Generation of haploid spermatids with fertilization and development capacity from human spermatogonial stem cells of cryptorchid patients. Stem Cell Reports. 2014; 3(4): 663-675, doi: 10.1016/j. stemcr.2014.08.004, indexed in Pubmed: 25358793.

38. Zhang S, Zeng Yi, Qu J, et al. Endogenous EGF maintains Sertoli germ cell anchoring junction integrity and is required for early recovery from acute testicular ischemia/ reperfusion injury. Reproduction. 2013; 145(2): 177-189, doi: 10.1530/REP-12-0336, indexed in Pubmed: 23241343. 